\title{
Unbiased least-squares modification of Stokes' formula
}

\author{
Lars E. Sjöberg ${ }^{1}$ (1) \\ Received: 1 February 2020 / Accepted: 23 July 2020 / Published online: 2 September 2020 \\ (c) The Author(s) 2020
}

\begin{abstract}
As the KTH method for geoid determination by combining Stokes integration of gravity data in a spherical cap around the computation point and a series of spherical harmonics suffers from a bias due to truncation of the data sets, this method is based on minimizing the global mean square error (MSE) of the estimator. However, if the harmonic series is increased to a sufficiently high degree, the truncation error can be considered as negligible, and the optimization based on the local variance of the geoid estimator makes fair sense. Such unbiased types of estimators, derived in this article, have the advantage to the MSE solutions not to rely on the imperfectly known gravity signal degree variances, but only the local error covariance matrices of the observables come to play. Obviously, the geoid solution defined by the local least variance is generally superior to the solution based on the global MSE. It is also shown, at least theoretically, that the unbiased geoid solutions based on the KTH method and remove-compute-restore technique with modification of Stokes formula are the same.
\end{abstract}

Keywords Modified Stokes formula $\cdot$ Remove-restore technique $\cdot$ Unbiased geoid estimation

\section{Introduction}

Today ultra-high Earth Gravitational Models (EGMs) allow detailed geoid determination all over the Earth. However, the higher-order harmonics of the EGMs are typically much less accurate than the low-to-medium wavelengths, which calls for improving the geoid estimator by using additional terrestrial gravity data in a combined local/regional solution. This is the case for some versions of the remove-compute-restore (RCR) method (e.g., Forsberg 1993; Sansò and Sideris 2013) and the least-squares modification of Stokes' formula (LSMSF; Sjöberg 1980, 1984a, b, 1991, 2003, 2005a). Due to truncations of the EGM series and the area of Stokes integration, such solutions have an inherent bias, and, in case of the LSMSF technique, the optimum solution is provided for the minimum of the expected global mean square error (MSE). However, Sjöberg (2005a) set forth the idea of optimizing the LSMSF method by minimizing the local MSE. While the LSMSF method implies that the EGM and gravity anomaly data are combined by spectral

Lars E. Sjöberg

larsjo@hv.se

1 Division of Mathematics, Computer and Surveying Engineering, University West (HV), 46186 Trollhättan, Sweden weighting at spherical harmonic degrees, Sjöberg (2011, Sect. 3) presented a method for local geoid determination by spectral combination and weighting by degree and order, being unbiased in case the truncation error is negligible. Similarly, Klees et al. (2018) tested the use of single- and multi-scale spherical radial base functions for local spectral combination of different sets of gravity-related data in geoid determination, but the result shows that the method needs further test and development before being suitable for practical application.

Here we will consider the LSMSF method in the case that Stokes' integral covers a sufficiently large region, such that the remote zone effect becomes negligible, in which case the solution can be regarded as unbiased (provided that all data are unbiased) from a statistical point of view. This implies that each solution (not necessarily the least-squares combination) will be (at least practically) unbiased, so that the MSE solution can be replaced by a local minimum variance solution.

Sjöberg and Bagherbandi (2017, Sect. 4.4.6) shortly discussed the possibility of modifying Stokes' formula with a very high-degree EGM, but the strategy was still to include the estimation bias in the model and minimizing the MSE. In contrast, here we will assume that the bias is negligible, implying that the minimum variance solution is optimal. Hence, both the unbiased LSMSF 
and Remove-Compute-Restore (RCR) solutions will be derived in Sects. 2 and 3, and the results are shortly discussed in Sect. 5. Section 6 concludes the study.

Although all of the above methods need direct and indirect corrections for topography, atmosphere and ellipsoidal shape of the Earth as well as for downward continuation of gravity observations to the sphere of integration, only the topographic effects are shortly discussed in Sect. 4 (but are more extensively treated in Sjöberg and Bagherbandi 2017, Sect. 6.2).

\section{The LSMSF solution}

\subsection{Spectral combination}

Consider the geoid height model

$$
\tilde{N}=\frac{c}{2 \pi} \iint_{\sigma} S^{L}(\psi) \Delta g^{T} \mathrm{~d} \sigma+c \sum_{n=2}^{L} s_{n} \Delta g_{n}^{\mathrm{EGM}},
$$

where $\sigma$ is the unit sphere, $c=R /(2 \gamma), \gamma$ being normal gravity on the reference ellipsoid, $R$ is the mean Earth radius, $\Delta g^{T}$ is the terrestrial gravity anomaly observation, $\Delta g_{n}^{\mathrm{EGM}}$ is the EGM derived gravity anomaly Laplace harmonic of degree $n$, and $L$ is the upper degree of the EGM as well as upper degree of the modification of Stokes' kernel function $S(\psi)$ by parameters $s_{k}$, i.e.:

$S^{L}(\psi)=S(\psi)-\sum_{k=2}^{L} \frac{2 k+1}{2} s_{k} P_{k}(\cos \psi)$,

where $P_{k}$ are Legendre's polynomials and $\psi$ is the geocentric angle of integration w.r.t. the computation point.

The spectral form of Eq. (1a) reads:

$\tilde{N}=c \sum_{n=2}^{\infty}\left(\lambda_{n}-s_{n}^{*}\right)\left(\Delta g_{n}+\varepsilon_{n}^{T}\right)+c \sum_{n=2}^{L} s_{n}\left(\Delta g_{n}+\varepsilon_{n}^{\mathrm{EGM}}\right)$,

where $\lambda_{n}=2 /(n-1), \Delta g_{n}$ is the true gravity anomaly, $\varepsilon_{n}^{T}$ and $\varepsilon_{n}^{\mathrm{EGM}}$ are the random errors of $\Delta g_{n}^{T}$ and $\Delta g_{n}^{E G M}$, respectively, and

$s_{n}^{*}= \begin{cases}s_{n}, & \text { if } n \leq L \\ 0 & \text { otherwise }\end{cases}$

Assuming that all error components are uncorrelated with vanishing expectations, and by subtracting the true geoid height
$N=c \sum_{n=2}^{\infty} \lambda_{n} \Delta g_{n}$

from Eq. (2a), the following error and variance of $\tilde{N}$ are obtained:

$\mathrm{d} \tilde{N}=c \sum_{n=2}^{\infty}\left(\lambda_{n}-s_{n}^{*}\right) \varepsilon_{n}^{T}+c \sum_{n=2}^{L} s_{n}^{*} \varepsilon_{n}^{\mathrm{EGM}}$

and

$\sigma_{\hat{N}}^{2}=E\left\{\mathrm{~d} \tilde{N}^{2}\right\}=c^{2} \sum_{n=2}^{\infty}\left(\lambda_{n}-s_{n}^{*}\right)^{2} \sigma_{n}^{2}+c^{2} \sum_{n=2}^{L} s_{n}^{2} \mathrm{~d} c_{n}^{2}$,

where $E\{\}$ is the statistical expectation operator, and $\sigma_{n}^{2}$ and $\mathrm{d} c_{n}^{2}$ are the error degree variances of the terrestrial and EGM gravity anomalies, respectively.

From Eq. (4), one notices that the geoid error is unbiased, and from Eq. (5) follows that the minimum variance related with the least-squares solution $(\hat{N})$ becomes

$\sigma_{\hat{N}}^{2}=c^{2} \sum_{n=2}^{L} \lambda_{n}^{2} \frac{\sigma_{n}^{2} \mathrm{~d} c_{n}^{2}}{\sigma_{n}^{2}+\mathrm{d} c_{n}^{2}}+c^{2} \sum_{n=L+1}^{\infty} \lambda_{n}^{2} \sigma_{n}^{2}$

for the modification parameters

$\hat{s}_{n}=\lambda_{n} \sigma_{n}^{2} /\left(\sigma_{n}^{2}+\mathrm{d} c_{n}^{2}\right)$.

The spectral weights given by Eq. (7) were first presented by Sjöberg (1980) and (1981) and by Wenzel (1981), who named this technique spectral combination.

We now generalize the initial geoid model (1a) by assuming that the terrestrial gravity and also the EGM errors are internally correlated with covariance matrices $\boldsymbol{\Sigma}$ and $\mathbf{C}$, respectively, (but gravity and EGM data are assumed as mutually uncorrelated). Then the geoid model (1a) is still unbiased, but now its variance becomes:

$\sigma_{\tilde{N}}^{2}=c^{2}\left(\lambda-\mathbf{s}^{*}\right)^{T} \boldsymbol{\Sigma}\left(\lambda-\mathbf{s}^{*}\right)+c^{2} \mathbf{s}^{T} \mathbf{C s}$,

or

$\sigma_{\tilde{N}}^{2}=c^{2} \lambda^{\mathrm{T}} \boldsymbol{\Sigma} \boldsymbol{\lambda}+c^{2} \mathbf{s}^{T}\left(\boldsymbol{\Sigma}_{L L}+\mathbf{C}\right) \mathbf{s}-2 c^{2} \lambda^{\mathrm{T}} \boldsymbol{\Sigma} \boldsymbol{s}^{*}$,

where we have decomposed the infinite matrix $\boldsymbol{\Sigma}$ into four parts with $\boldsymbol{\Sigma}_{L L}$ and $\boldsymbol{\Sigma}_{\infty \infty}$ on the diagonal and $\boldsymbol{\Sigma}_{L \infty}=\boldsymbol{\Sigma}_{\infty L}^{T}$ off-diagonal, of dimensions $(L, L),(\infty, \infty)$ and $(L, \infty)$, respectively. (In practice, the infinite dimensions must be approximated by some finite degree.) Differentiating $\sigma_{\tilde{N}}^{2}$ w.r.t. $\mathbf{s}$ and equating to zero one arrives at the least-squares choice of $\mathbf{s}$ :

$\left(\boldsymbol{\Sigma}_{L L}+\mathbf{C}\right) \mathbf{s}-\boldsymbol{\beta}=\mathbf{0} \Rightarrow \hat{\mathbf{s}}=\left(\boldsymbol{\Sigma}_{L L}+\mathbf{C}\right)^{-1} \boldsymbol{\beta}$,

where 
$\boldsymbol{\beta}=\left(\boldsymbol{\Sigma}_{L L} \boldsymbol{\Sigma}_{L \infty}\right) \boldsymbol{\lambda}$,

with the variance of the geoid height estimator

$\sigma_{\hat{N}}^{2}=c^{2}\left[\lambda^{\mathrm{T}} \boldsymbol{\Sigma} \lambda-\hat{\mathbf{s}}^{\mathrm{T}} \boldsymbol{\beta}\right]=c^{2}\left[\lambda^{\mathrm{T}} \boldsymbol{\Sigma} \lambda-\hat{\mathbf{s}}^{T}\left(\boldsymbol{\Sigma}_{L L}+\mathbf{C}\right) \hat{\mathbf{s}}\right]$.

\subsection{Solutions for a small integration cap size}

We now allow for a high-degree maximum $(M)$ of the EGM, such that $M \geq L$, and we limit the Stokes integration in Eq. (1a) to a cap $\sigma_{0}$ of spherical radius $\psi_{0}$. Then the general estimator becomes:

$\tilde{N}=\frac{c}{2 \pi} \iint_{\sigma_{0}} S^{L}(\psi) \Delta g^{T} d \sigma+c \sum_{n=2}^{M}\left(Q_{n}^{L}+s_{n}^{*}\right) \Delta g_{n}^{\mathrm{EGM}}$,

where

$Q_{n}^{L}=Q_{n}^{L}\left(\psi_{0}\right)=\int_{\psi_{0}}^{\pi} S^{L}(\psi) P_{n}(\cos \psi) \sin \psi \mathrm{d} \psi=Q_{n}-\sum_{k=2}^{L} E_{n k} S_{k}$

and

$Q_{n}=\int_{\psi_{0}}^{\pi} S(\psi) P_{n}(\cos \psi) \sin \psi \mathrm{d} \psi$

are the so-called Molodensky truncation coefficients with

$E_{n k}=E_{n k}\left(\psi_{0}\right)=\frac{2 k+1}{2} \int_{\psi_{0}}^{\pi} P_{n} P_{k} \sin \psi \mathrm{d} \psi$.

In this case, the geoid estimator can be written in the spectral form as

$\tilde{N}=c \sum_{n=2}^{\infty}\left(\lambda_{n}-Q_{n}^{L}-s_{n}^{*}\right)\left(\Delta g_{n}+\varepsilon_{n}^{T}\right)+c \sum_{n=2}^{M}\left(Q_{n}^{L}+s_{n}^{*}\right)\left(\Delta g+\varepsilon_{n}^{\mathrm{EGM}}\right)$,

and the geoid height error becomes

$$
\begin{aligned}
\mathrm{d} N= & c \sum_{n=2}^{\infty}\left(\lambda_{n}-Q_{n}^{L}-s_{n}^{*}\right) \varepsilon_{n}^{T} \\
& +c \sum_{n=2}^{M}\left(Q_{n}^{L}+s_{n}^{*}\right) \varepsilon_{n}^{\mathrm{EGM}}-c \sum_{n=M+1}^{\infty} Q_{n}^{L} \Delta g_{n} .
\end{aligned}
$$

Here it will be assumed that $M$, the maximum degree of the EGM, is so large, that the bias, the last term of Eq. (12), is negligible at the $\mathrm{cm}$-level already for integral cap sizes of a few degrees (see Note 1 at the end of this section). Sjöberg (2005b, Sect. 5) reported that by selecting the modification parameters according to Molodensky's method (Molodensky et al. 1962), which is specifically designed to minimize the truncation error, with $L=M=360$, the RMS truncation error is within $1 \mathrm{~mm}$ already for a cap size of $2^{\circ}$. However, in the LSMSF technique the bias will be larger as it is balanced with other error components in a minimum MSE solution.

Disregarding the bias and assuming that the errors of $\Delta g^{T}$ and $\Delta g^{\mathrm{EGM}}$ are uncorrelated, the following variance of the general geoid estimator of Eq. (10a) follows:

$$
\begin{aligned}
\sigma_{\tilde{N}}^{2}= & c^{2}\left[(\boldsymbol{\lambda}-\mathbf{q}+\mathbf{D} \mathbf{s})^{T} \mathbf{\Sigma}(\boldsymbol{\lambda}-\mathbf{q}+\mathbf{D} \mathbf{s})\right. \\
& \left.+\left(\mathbf{q}_{M}-\mathbf{D}_{M} \mathbf{s}\right)^{T} \mathbf{C}\left(\mathbf{q}_{M}-\mathbf{D}_{M} \mathbf{s}\right)\right],
\end{aligned}
$$

where the infinite number of elements of vector $\mathbf{q}$ are $q_{n}=Q_{n}\left(\psi_{0}\right), \mathbf{D}$ and $\mathbf{E}$ are matrices of dimensions $(\infty, L)$ with $\mathbf{D}=\mathrm{E}-(\mathbf{I} \mathbf{0})^{T}, \mathbf{E}$ with elements $E_{n k}$ and $\mathbf{I}$ being a unit matrix of dimension $(L, L)$, and $\mathbf{q}_{M}$ and $\mathbf{D}_{M}$ are the first $M$ rows of $\mathbf{q}$ and $\mathbf{D}$.

The least-squares choice of the modification parameters $\mathbf{s}$ can be obtained by differentiating the variance of Eq. (13) by $\mathbf{s}$ and equating to zero. The result is:

$(\boldsymbol{\lambda}-\mathbf{q}+\mathbf{D s})^{T} \mathbf{\Sigma} \mathbf{D}-\left(\mathbf{q}_{\mathbf{M}}-\mathbf{D}_{M} \mathbf{s}\right)^{T} \mathbf{C D}_{M}=\mathbf{0}$,

or

As $=\mathbf{h}$,

where

$\mathbf{A}=\left[\mathbf{D}^{T} \Sigma \mathbf{D}+\mathbf{D}_{M}^{T} \mathbf{C D}_{M}\right]$ and $\mathbf{h}=\mathbf{D}_{M}^{T} \mathbf{C} \mathbf{q}_{M}-\mathbf{D}^{T} \boldsymbol{\Sigma}(\boldsymbol{\lambda}-\mathbf{q})$,

and the solution for $\mathbf{s}$ becomes:

$\hat{\mathbf{s}}=\mathbf{A}^{-1} \mathbf{h}$.

Finally, by substituting $\hat{\mathbf{s}}$ for $\mathbf{s}$ in Eq. (13), one arrives at the following variance for the least-squares geoid estimator:

$\sigma_{\hat{N}}^{2}=f-c^{2} \hat{\mathbf{s}}^{\mathrm{T}} \mathbf{h}=f-\mathbf{h}^{T} \mathbf{A}^{-1} \mathbf{h}$,

where

$f=c^{2}\left[(\boldsymbol{\lambda}-\mathbf{q})^{T} \boldsymbol{\Sigma}(\boldsymbol{\lambda}-\mathbf{q})+\mathbf{q}_{M}^{T} \mathbf{C} \mathbf{q}_{\mathrm{M}}\right]$.

Note 1 The significance of the bias term in Eq. (12) can be controlled by the approximation

$\left|\mathrm{d} N_{\text {bias }}\right| \approx c \sqrt{\sum_{n=M+1}^{\infty}\left(Q_{n}^{L}\right)^{2} c_{n}}$

where $c_{n}$ are the components of a gravity anomaly (signal) degree variance model. 
Note 2 If the aim were to find the minimum MSE solution, the square of the bias must be added to the variance in Eq. (13) for differentiation w.r.t. s. However, this is not the goal of this study.

\section{The RCR solution}

Using the remove-compute-restore technique, the geoid estimator corresponding to Eq. (1a), where $L=M$, can be written

$\tilde{N}=\frac{c}{2 \pi} \iint_{\sigma} S^{L}(\psi)\left(\Delta g^{T}-\Delta g^{\mathrm{EGM}}\right) \mathrm{d} \sigma+c \sum_{n=2}^{L} \lambda_{n} \Delta g_{n}^{\mathrm{EGM}}$,

where

$\Delta g^{\mathrm{EGM}}=\sum_{n=2}^{L} \Delta g_{n}^{\mathrm{EGM}}$,

and the spectral form of this estimator becomes effect on the Molodensky type of surface gravity anomalies ( $\left(\Delta g^{T}\right.$ and $\left.\Delta g^{\mathrm{EGM}}\right)$. Then the gravity anomalies are downward continued in one way or another to the sphere of computation, a secondary indirect topographic effect on $\Delta g^{T}$ is applied, and after Stokes integration, the first indirect topographic effect on the geoid is added (e.g., Sjöberg 2018). The interested reader is also referred to Sjöberg and Bagherbandi (2017, Sect. 6.2) to find that also these corrections theoretically agree in the KTH and RCR approaches when properly applied. See also Sjöberg (2005b).

\section{Discussion}

It must be emphasized that the above modification parameters and least-squares solutions are local, implying that the modification parameters change with position on the mean Earth sphere. However, in practice, one can expect small changes in a small region, implying that only one set of modification parameters is needed there. As all solutions are unbiased, any choice of modification parameters yields

$\tilde{N}=c \sum_{n=2}^{L}\left(\lambda_{n}-s_{n}\right)\left(\Delta g_{n}^{T}-\Delta g_{n}^{\mathrm{EGM}}\right)+c \sum_{n=L+1}^{\infty} \lambda_{n} \Delta g_{n}^{T}+c \sum_{n=2}^{L} \lambda_{n} \Delta g_{n}^{\mathrm{EGM}}$,

which, after some rearrangements of terms, equals Eq. (2a).

Similarly, using the spectral representation, the more general Eq. (10a) for the KTH approach with $L \leq M$ can be rewritten in its RCR- form as

$\tilde{N}=\frac{c}{2 \pi} \iint_{\sigma_{0}} S^{L}(\psi)\left(\Delta g^{T}-\Delta g^{\mathrm{EGM}}\right) \mathrm{d} \sigma+c \sum_{n=2}^{M} \lambda_{n} \Delta g_{n}^{\mathrm{EGM}}$.

That is, from a theoretical point of view the RCR technique yields the same set of estimators as the straightforward modification of Stokes' formula. In particular, the least-squares modification parameters and geoid variance become the same for the two methods.

\section{Topographic corrections}

As stated at the end of Sect. 1, the corrections for topography are treated only sparsely in this study. The KTH approach uses analytical continuation of the external disturbing potential to point level and geoid level in applications for quasiand geoid determination, respectively, and there is no further topographic correction in quasigeoid estimation, while geoid estimates need also corrections for the topographic bias. In the RCR method, firstly one removes the effect of the topography on the observables as the direct topographic an unbiased solution (if the truncation bias vanishes). Hence, the weight relation between terrestrial and EGM gravity data will not be critical for the solution, but, as is known from any least-squares adjustment, the error estimation will be more dependent on the choice of error covariance models. An important advantage of the unbiased solutions is that the gravity anomaly signal degree variance model needed in the MSE solutions is not required.

A similar approach for geoid determination was outlined in Sjöberg (2005a), but the principle difference is that there the geoid bias term was included in the optimization, which leads to a least-squares solution based on the (local) MSE. Hence, if the bias term is small, the two solutions should be practically the same.

\section{Concluding remarks}

Unbiased minimum variance estimators are usually preferred to biased/MSE solutions, demanding less a priori information for practical applications. The spectral combination without assuming correlations is the most simple solution, but not very realistic as there are mostly correlations among the data that should be taken into account. Doing so in the strict sense lead to huge, even infinite dimensional, covariance matrices, which, of course, in practice must 
be restricted to a suitable resolution/dimension. Here one should also remember that approximate/simplified covariance models also lead to unbiased, even if not optimal, solutions.

We have shown that the unbiased LSMSF and RCR solutions are theoretically the same, although there may be some differences in their practical implementations.

Acknowledgements Open access funding was provided by University West. This study was supported by Project No. 187/18 of the Swedish National Space Agency (SNSA).

Open Access This article is licensed under a Creative Commons Attribution 4.0 International License, which permits use, sharing, adaptation, distribution and reproduction in any medium or format, as long as you give appropriate credit to the original author(s) and the source, provide a link to the Creative Commons licence, and indicate if changes were made. The images or other third party material in this article are included in the article's Creative Commons licence, unless indicated otherwise in a credit line to the material. If material is not included in the article's Creative Commons licence and your intended use is not permitted by statutory regulation or exceeds the permitted use, you will need to obtain permission directly from the copyright holder. To view a copy of this licence, visit http://creativecommons.org/licenses/by/4.0/.

\section{References}

Forsberg R (1993) Modelling the fine-structure of the geoid: methods, data requirements and some results. Surv Geophys 14:403-418

Klees R, Slobbe DC, Farahani HH (2018) A methodology for leastsquares local quasi-geoid modelling using a noisy satellite-only gravity field model. J Geod 2018(92):431-442. https://doi. org/10.1007/s00190-017-1076-0

Molodensky MS, Eremeev VF, Yurkina MI (1962) Methods for study of the external gravitational field and figure of the Earth. Trans from Russian by Israel Programme for Scientific Translations, Jerusalem

Sansò F, Sideris MG (eds) (2013) Geoid determination-theory and methods. Springer, Berlin. https://doi.org/10.1007/978-3-540$74700-0$

Sjöberg LE (1980) Least squares combination of satellite harmonics and integral formulas in physical Geodesy. Gerlands Beiträge zur Geophysik 89(5):371-377

Sjöberg LE (1981) Least squares combination of satellite and terrestrial data in physical geodesy. Ann Geophys 37:25-30

Sjöberg LE (1984a) Least-Squares modification of Stokes' and VeningMeinez' formula by accounting for truncation and potential coefficients errors. Manusc Geod 9:209-229

Sjöberg LE (1984b) Least squares modification of Stokes' and Vening Meinesz' formulas by accounting for errors of truncation, potential coefficients and gravity data. Department of Geodesy Rep No 27, University of Uppsala, Uppsala, Sweden

Sjöberg LE (1991) Refined least-squares modification of Stokes' formula. Manus Geod 16:367-375

Sjöberg LE (2003) A general model for modifying Stokes' formula and its least-squares solution. J Geod 77:459-464

Sjöberg LE (2005a) A local least-squares modification of Stokes' formula. Stud Geophys Geod 49:23-30

Sjöberg LE (2005b) A discussion on the approximations made in the practical implementation of the remove-compute-restore technique in regional geoid modelling. J Geod 78:645-653

Sjöberg LE (2011) Local least squares spectral filtering and combination by harmonic functions on the sphere. J Geod Sci 1:355-360

Sjöberg LE (2018) Topographic effects in geoid determinations. Geosciences 8:143. https://doi.org/10.3390/geosciences8040143

Sjöberg LE, Bagherbandi M (2017) Gravity Inversion and Integrationtheory and applications in geodesy and geophysics. Springer, Berlin

Wenzel H-G (1981) Zur Geoidbestimmung durch Kombination von Schwereanomalien und einem Kugelfunktionsmodell mit Hilfe von Integralformeln. Z. Vermess 106:102-111 